Volume 8, No. 7, July - August 2017

International Journal of Advanced Research in Computer Science

RESEARCH PAPER

Available Online at www.ijarcs.info

\title{
TRUST BASED MULTI PATH ROUTING \& TOPOLOGY HIDING IN MOBILE AD HOC NETWORK : A SECURE PERSPECTIVE
}

\author{
Mr. Rahul K Ambekar \\ Research Scholar, Pacific Academy of Higher Education and \\ Research University, Udaipur (Rajasthan)313003
}

\author{
Dr. Uttam D. Kolekar \\ Professor \& Principal \\ A.P.Shah Institute of Technology \\ Thane West, Thane, Maharashtra 400615
}

\begin{abstract}
Mobile Ad-hoc Network (MANET) has been atracted large number of researchers in recent years due to its inherent potential for the data transmission .The peculiar charectristics of MANET like limited battery power, mobility of nodes, limited bandwidth provides challenge for the effective and efficient routing among the mobile nodes and at the same time it is necessary to ensure secure data transmission within the given constraints of MANET . Multipath routing is much more flexible and effective approach of routing . Topology hiding protocol i.e. TOHIP resolves the topology exposure problem im multi path routing. FL-TOHIP \& AFL-TOHIP has demostrated the improvement in solution searching and enhanced the efficiency of TOHIP . Malicious nodes and other attacks make MANET vulnerable even with topology hiding . Trust based aapproach is used to secure communication using the trust between the neighbours . Trust based approach is an alternative to cryptographic tecniques. This paper evaluates variois trust based protocols and provide a direction to integrate trust based approach with topology hiding multi path routing protocols in MANET to provide optimal data transmission with utmost security .
\end{abstract}

Keywords : MANET, Multi path routing, Topology hiding, Trust

I. Introduction : Mobile ad hoc network (MANET) is a self organising wireless network of mobile nodes. The MANET is an autonomus network with temporary topologies. The strikimg application of such network is in critical situations like rescue operations, military intelligence, natural calamities where the possibility of existing network or fixed infrastructure is negligible. The peculiar charecteristics of MANET is that nodes are mobile and they completely survive on battery . Since external source of power is not possible, the nodes are required to use their battery judiciousy. Nodes in MANET also share limited bandwidth. Due to mobility of nodes the probability of failiure of data transmission increases significantly . Routing in such constraints is the most vital strategy to improve reliable and efficient data transmission .

Several routing strategies and protocols based on those routing strategies have been explored by the researchers. The primary classification of routing methods can be done as single path routing and multi path routing. The routing protocols are also classified as proactive , reactive and hybrid protocols . Proactive protocols are also called as table driven protocols . Reactive protocols are called as demand driven protocols . Hybrid protocols are the combination of proactive and reactive protocols .

Section II of this paper present the overview of multi path routing \& topology hiding. Section III of this paper discuccses related work of various aspects of trust based MANET . Section IV of this paper propose integration of topology hiding \& trust based approach. Section V concludes the paper .

II. Overview of Multi Path Routing protocols \& topology hiding : The Unipath routing protocols uses only one path for the routing between source \& destination. The multi path routing protocols consists of finding multiple routes between source and destination . It offerers several benefits like load balancing, fault tolerance, higher aggregate bandwidth, lower end to end delay[1] . Multi path protocols are broadly classified into five categories based on the goals. The goals are to improve delay, provode reliability, reduce overhead, maximise network life and hybrid routing [2]. Several challenges and peculiar issues with regard to multi path routing in Manet are discussed in [3]. Load balancing is one of

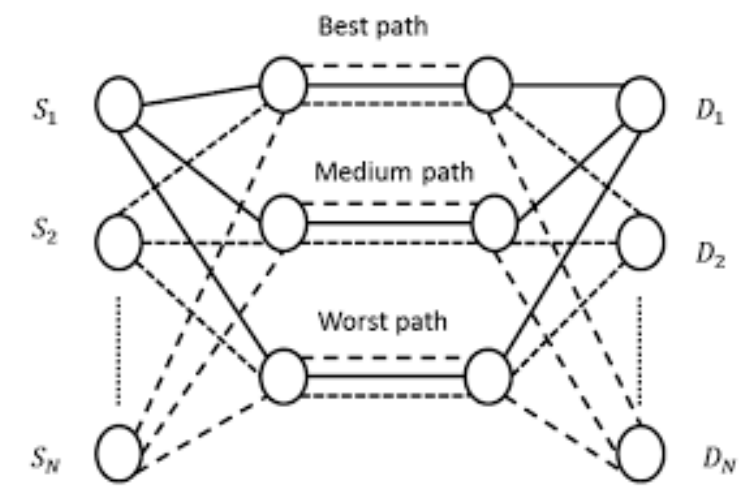

the most vital constraints in mobile ad hoc network due to limited bandwidth . To provide a trustworthy quality service guarranty in mobile ad hoc network is very much challenging [4]. With the use of multiple paths for the communication between source \& destination , the automatic user becomes almost unaware of the possible network failiure due to security attacks or link collapses [5].

Fig 1 : Illustration of Multi Path Routing

Multi path routing protocols offeres several benefits over unicast routing but they are vulnerable to various security threats due to different comstraints . Topology exposure problem in multipath routing protocol exposes the entire topology between possible pairs of source and destination 
whilie routing . Topology exposure mayt threaten security in multi path routing protocols in Manet. [6] Topology Hiding protocol eliminates the problem of topology exposure problem by hiding topologies while routing through multiple paths [7] . FL-TOHIP \& AFL-TOHIP protocols improved the efficiency \& throughput of topology hiding multipath routing protocol[8][9].

\section{Trust based Multi path Routing Protocols in Manet :} Due to multi -hop routing and absense of centralised administration Manets are velnerable to several attacks [10] . The Manet with multi path routing is more prone to said attacks . A node trust and continued path trust is used to discover trustworthy paths and reduce attacks from malicious nodes [10]. Several trust based models and protocols are discovered to secure data transmission by reducing the possible attacks . Light weight IDS takes care of black hole attack \& grey hole attack.Light weight ttrust based protocol 1 is used to reduce overhead and increase scalability[11]. The depolyment of any security policy requires the definition of trust model that defines wo trusts who and how . Certification based model with public service key is used to secure Manret [12]. The purpose of secure and trust based on demand multi path routing is to find trust based secure routes between source and destination. The mesh based multi path routing scheme in [13] is used to discover all possiible secure paths using adjacent trust verification . Trust and reputation mechanism is used in [14] to improve link quality and channel utilisation. Trust mechanism is prefered over cryptographic techniques The trust mechanism secures data forwarding by isolating nodes with malicious intentions using trust value on the nodes[15].

\section{Praposed Integration of Topology Hiding \& Trust} Basedopology Routing for Muliti Path Routing inManet : Topology hiding overcomes the problem of topology exposure problem while routing the transmission between source and destination but though it eliminates some of the attacks , efficiency reduces under attacks . The purpose of effcient transmission in Manet with constrained resources like limited bandwidth, limited power and mobile nodes is to increase the lifetime of network through efficient and effective routing . Several attacks and malicious nodes threaten the security of network and may reduce the network life time in Manet . Multi Path routing compared to single path routing offeres more flexibility and more probability of succesful transmission in the mobile enviornment of Manet with given limited resources and constraints. To increase network life time and enhance security against all possible attacks and the problem of malicious nodes we propose the integration of topology hiding and trust based multi path routing in Manet. The proposed integration will escalate the securty at two levels Viz. $1^{\text {st }}$ level : Due to hiding of topology the complete path between source and destination will be hidden and hence it will be difficult for the attackers to intrude or evasdrop the communication $.2^{\text {nd }}$ level: Trust mechanism will allow to choose reliable neighbors based on the trust established. The reliable nodes helps to choose the optimal and secure path among multiple paths in multi path routing . The integration of topology hiding and trust based routing is sought to reduce end to end delay, reduce packet drop, increase througput with utmost securty from all possible attacks. The proposed integrated topology hiding and trust based protocol intends to use four phase aproach i.e. [1] Route creation at the source .[2] Route creation at the intermediate nodes . [3] Route creation at the destination node .

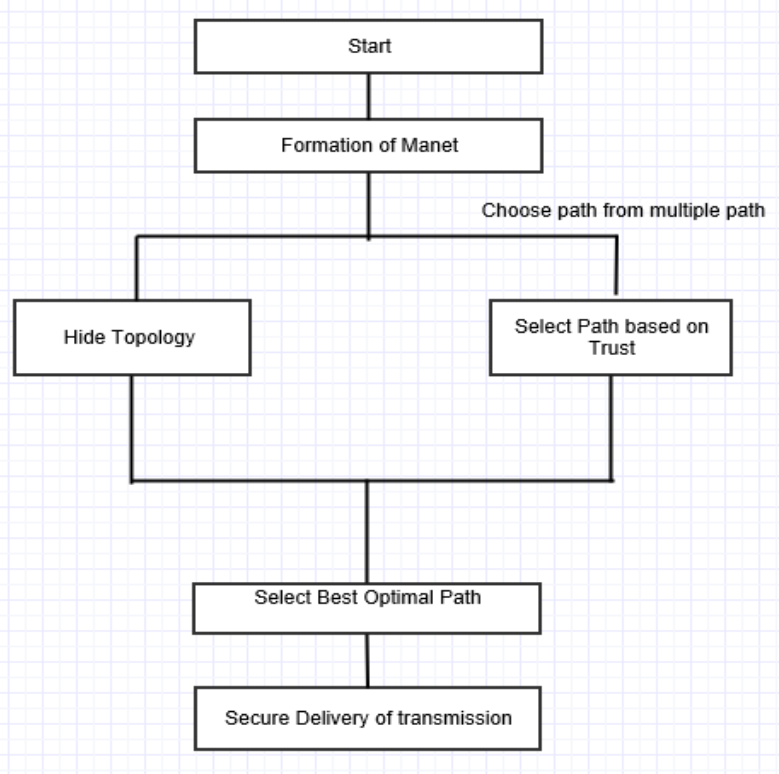

Fig.2 : Proposed Block Diagram of integration of topology hiding amd trust based routing .

V. Conclusiom : In this paper we have discussed the multi path routing protocols in Manet . We have discussed flexibility and benefits of multi path routing over single path routing in Manet with restricted resources and constraints . The exposure topology while multi path routing may expose yse the network for the attackers with greater security risc . Topology hiding protocols eliminates the prob;lem of topology exposure but they are not sufficient to meet all secuirty precautions . We furthure discuseed the trust based routing to improve security against various attacks. In this paper we have proposed two level security approach integrating topology hiding \& trust based routing using multi path routing in Manet. The proposed work intends to increase falut tolerent data transmission by allowing the capabilities of multi path routing and securing the data transmission with topology hiding and trust based routing among the multiple paths .

\section{REFERENCES:}

[1] YI Jiazi, “ Sumary of Routong protocols in Mobile Ad-Hoc Network”, Polytechnic School of University of Nantes , March 2007.

[2] Mohammed Tarique, Kemal E. Tepe, Sasan Adibi, Shervin Erfani, " Survey of multi path routing protocols for mobile ad hoc networks", Journal of Computer and Network Applications , Volume 32, issue 6, November 2009, pages 1125-1143.

[3] Stephen Mueller, Rose . P.Tsang .et ,” Multi Path Routing in Mobile Ad Hoc Networks : Issues \& Challenges.”, Sandia National Laboratories, CA,USA, 2003.

[4] Yogesh Chaba, R.B. Patel et. , “ Issues and challenges involved in multi path routing with dymo protocol”, International Journal of Information Technology and Knowledge Management , January-Jume2012, Volume 5,No.1pp 21-25. 
[5] Rosa Movropadi, Christos Douligeris, “ Multi Path Routing for Mobile Ad Hoc Networks : Issues \& Performance Evaluation.”, WAC 2005, pp 165-176.

[6] Yujun Zhang et. , “ Design and performance study of Topology Hiding Multi Path Routing protocol for Mobile Ad Hoc Networks”, 2012 IEEE INFOCOM Proceedings .

[7] Yujun Zhang et, "TOHIP: A Topology Hiding Multi Path Routing protocol for Mobile Ad Hoc Networks” Ad Hoc Networks 21(2014) 109-122.

[8] Rahul Ambekar, Uttam Kolekar, “ FL-TOHIP: Fractional Lion Optimisation to Topology Hiding Multi Path Routing in Mobile Ad Hoc Networks” Journal of Intelligent Systems, 2017 .

[9] ]Rahul Ambekar, Uttam Kolekar, “ AFL-TOHIP: Adaptive Fractional Lion Optimisation to Topology Hiding Multi Path Routing in Mobile Ad Hoc Networks” ISMAC, IEEE International Conference Palladam Tamilnadu 2017.

[10] X.Li., P.Zhang et., “ Trust-based on-demand Multi Path Routing in Mobile Ad Hoc Networks”, IET Information Security.2010.ISSN 1751-8709.
[11] N.Marchang, Rdatta, “ Light-Weight Trust-based routing protocol for Mobile Ad Hoc Networks”, IET Information Security 2011, ISSN 1751-8709.

[12] Mawlond Omar et., " Certification based trust models in Moblle Ad Hoc Networks: A Survey and Taxonomy”, Journal of Network and Computer Applications 35(2012) 268-286.

[13] Gautam Borkar, A.R.Mahajan, “ A Secure and trust-based on demand multi path routing scheme for self organised mobile ad hoc networks”, Wireless Network, Springer Media 2016.

[14] Ramya Dorai, M Rajaram, “ Trust and Reputation Mechanism with Path Optimisation in Multipath Routing “, International Journal of Electrical ,

Computer,Energitic,Electronic and Communication Engineering Vol 9 , No 3,2015.

[15] Mousami Sardar, Kaushik Majumdar, “ A Comparative Study on different trust based routing schemes in Manet”, International Journal of Wireless \& Mobile Networks Vol 5,No 5, October 2013. 